\title{
ON TRIPLE SEQUENCE OF BERNSTEIN OPERATOR OF WEIGHTED ROUGH $I_{\lambda}$-CONVERGENCE
}

\author{
NAGARAJAN Subramanian AND AYHAN ESI
}

Abstract. We introduce and study some basic properties of rough $I_{\lambda}$-convergence of weight $g$, where $g: \mathbb{N}^{3} \rightarrow[0, \infty)$ is a function satisfying $g(m, n, k) \rightarrow \infty$ and $\frac{\|(m, n, k)\|}{g(m, n, k)} \nrightarrow 0$ as $m, n, k \rightarrow \infty$, of triple sequence of Bernstein polynomials and also study the set of all rough $I_{\lambda}$-convergence of weight $g$ limits of a triple sequence of Bernstein polynomials and relation between analyticness and rough $I_{\lambda}$-convergence of weight $g$ of a triple sequences of Bernstein polynomials.

Mathematics subject classification (2010): 40F05, 40J05, 40G05, $40 \mathrm{G} 15$.

Keywords and phrases: Triple sequences, rough convergence, closed and convex, cluster points and rough limit points, Bernstein polynomials, $I$-convergence.

\section{REFERENCES}

[1] S. Aytar, Rough statistical convergence, Numer. Funct. Anal. Optimiz. 29 (3-4) (2008) 291-303.

[2] S. AYTAR, The rough limit set and the core of a real sequence, Numer. Funct. Anal. Optimiz. 29 (3-4) (2008) 283-290.

[3] M. BAlcerzak, P. DAs, M. Filipczak, AND J. SwACZYNA, Generalized kinds of density and the associated ideals, Acta Math. Hungar. 147 (1) (2015) 97-115.

[4] H. CARTAN, Filters et ultrafilters, C. R. Acad. Sci. Paris 205 (1937) 777-779.

[5] A. J. DutTA, A. Esi, B. C. TRIPATHy, Statistically convergent triple sequence spaces defined by Orlicz function, J. Math. Anal. 4 (2) (2013) 16-22.

[6] S. Debnath, B. SARMA, B. C. DAs, Some generalized triple sequence spaces of real numbers, J. Nonlinear Anal. Optimiz. 6 (1) (2015) 71-79.

[7] E. DÜNDAR, C. ÇAKAN, Rough I-convergence, Demonstratio Math. XLVII (3) (2014) 638-651.

[8] E. DÜNDAR, C. ÇAKAN, Rough convergence of double sequences, Gulf J. Math. 2 (1) (2014) 45-51.

[9] E. DündAR, On Rough $\mathscr{I}_{2}$-Convergence of Double Sequences, Numer. Funct. Anal. Optimiz. 37 (4) (2016) 480-491.

[10] A. ESI, On some triple almost lacunary sequence spaces defined by Orlicz functions, Research and Reviews: Discrete Math. Structures 1 (2) (2014) 16-25.

[11] A. Esi, M. Necdet Catalbas, Almost convergence of triple sequences, Global J. Math. Anal. 2 (1) (2014) 6-10.

[12] A. ESI, E. ŞAVAS, On lacunary statistically convergent triple sequences in probabilistic normed space, Appl. Math. Inf. Sci. 9 (5) (2015) 2529-2534.

[13] A. Esi, S. Araci, M. AcIKGoz, Statistical Convergence of Bernstein Operators, Appl. Math. Inf. Sci. 10 (6) (2016) 2083-2086.

[14] A. EsI, $\lambda_{3}$-Statistical convergence of triple sequences on probabilistic normed space, Global J. Math. Anal. 1 (2) (2013) 29-36.

[15] B. HazArika, S. A. Mohiuddine, Ideal convergence of random variables, J. Func. Spaces Appl. 2013 (2013), Article ID 148249, 7 pages.

[16] P. Kostyrko, T. S̆Alát, W. Wilczyśki, On I-convergence, Real Analysis Exchange 26 (2) (2000-2001) 669-686.

[17] P. MALIK, M. Maity, On rough statistical convergence of double sequences in normed linear spaces, Afr. Mat. 27 (1) (2016) 141-148. 
[18] F. NURAY, W. H. RUCKLE, Generalized statistical convergence and convergence free spaces, J. Math. Anal. Appl. 245 (2000) 513-527.

[19] S. K. Pal, D. Chandra, S. Dutta, Rough Ideal Convergence, Hacettepe J. Math. Stat. 42 (6) (2013) 633-640.

[20] H. X. PHU, Rough convergence in normed linear spaces, Numer. Funct. Anal. Optimiz. 22 (2001) 199-222.

[21] H. X. PHU, Rough continuity of linear operators, Numer. Funct. Anal. Optimiz. 23 (2002) 139-146.

[22] H. X. PHU, Rough convergence in infinite dimensional normed spaces, Numer. Funct. Anal. Optimiz. 24 (2003) 285-301.

[23] A. Sahiner, M. Gurdal, F. K. Duden, Triple sequences and their statistical convergence, Selcuk J. Appl. Math. 8 (2) (2007) 49-55.

[24] A. Sahiner, B. C. TRipathy, Some I related properties of triple sequences, Selcuk J. Appl. Math. 9 (2) (2008) 9-18.

[25] T. Šalát, B. C. Tripathy, M. Ziman, On some properties of I-convergence, Tatra Mt. Math. Publ. 28 (2004) 279-286.

[26] E. SaVAS, A. ESI, Statistical convergence of triple sequences on probabilistic normed space, Ann. Uni. Craiova, Math. Compu. Sci. Series 39 (2) (2012) 226-236.

[27] B. C. Tripathy, R. Goswami, On triple difference sequences of real numbers in probabilistic normed spaces, Proyecciones J. Math. 33 (2) (2014) 157-174.

[28] A. ESI, S. ARACI AND A. EsI, $\lambda$-Statistical convergence of Bernstein polynomial sequences, Advances and Application in Mathematical Sciences, 16 (3) (2017), 113-119.

[29] A. ESI, N. SUBRAMANIAN AND A. ESI, Triple rough statistical convergence of sequence of Bernstein operators, Int. J. Adv. Appl. Sci. 4 (2) (2017), 28-34.

[30] B. C. TRIPathy AND R. Goswami, Vector valued multiple sequences defined by Orlicz functions, Boletim da Sociedade Paranaense de Matematica, 33 (1) (2015), 67-79.

[31] B. C. TRIPATHY AND R. Gos WAMI, Multiple sequences in probabilistic normed spaces, Afrika Mathematica, 26 (5-6) (2015), 753-760.

[32] B. C. TRIPATHY AND R. Gos WAMI, Fuzzy real valued p-absolutely summable multiple sequences in probabilistic normed spaces, Afrika Mathematica 26 (7-8) (2015), 1281-1289. 